Schwefelarsensäure von $\mathrm{Cloez}$ haben wir schon ähnliche Beispicle. Schliefslich will ich die erwähnten analogen Verbindungen, der Uebersicht wegen zusammenstellen :

Kohlensaures Ammoniumoxyd $\left.2 \mathrm{C}_{4} \mathrm{H}_{4} \mathrm{O}, \mathrm{CO}_{2}\right)$,

Carbaminsaures Ammoniunoxyd $\mathrm{N} \mathrm{H}, \mathrm{O}, \mathrm{C} \mathrm{N} \mathrm{H}_{2} \mathrm{O} ; \mathrm{CO}_{2}$,

Cyansaures Ammoniumoxyd $\quad \mathrm{N} \mathrm{H}_{4} \mathrm{O}, \mathrm{C}_{2} \mathrm{~N} \mathrm{O}$,

Ammoniumsulfocarbonat $2\left(\mathrm{~N} \mathrm{H}_{4} \mathrm{~S}, \mathrm{CS}_{2}\right)$,

Sulfocarbamins. Ammoniumsulfuret $\mathrm{N} \mathrm{H}_{4} \mathrm{~S}, \mathrm{CN} \mathrm{H}_{2} \mathrm{~S} ; \mathrm{CS}_{2}$,

Schwefelcyanammonium $\quad \mathrm{N} \mathrm{H}_{4} \mathrm{~S}, \mathrm{C}_{2} \mathrm{NS}$.

\title{
Ueber die Natur des metallischen Tilans;
}

\section{von $F$. Wöller.}

(Aus den Abhandlungen der K, Gesellschaft d. Wissenschaften zu Göttingen.)

Die schönen kupferfarbenen Würfel von Titan, deren Bildung in den Hohöfen so häufig beobachtet wird, sind nicht das, wofür man sic bis jelzt gchalten hat, sie sind nicht ein einfacher, sondern sie sind ein zusammengesetzter Körper. Sie bestchen aus einer Verbindung von Cyantitan mit Stickstofftitan, zusammengesetzt nach der Formel : $\mathrm{Ti}^{2} \mathrm{~N}+3 \mathrm{Ti}^{3} \mathrm{~N}$. Diese Zusammensetzung wird durch die folgenden Thalsachen bewiesen :

Erhitzt man die Würfel in trocknem Chlorgas, so bildet sie bekanntlich liquides 'Titanclılorid, zugleich aber sublimirl sich in reichlicher Menge ein sehr fliichtiger Körper in kleinen, schwefelgelben Krystallen. Diese Krystalle sind eine Verbindung von Tilanchlorid mit Cyanchlorid. Von Wasser werden sie unler Erhilzung aufgelösl und diese Lösung besitzt, nachdem man durch Quecksilber das freie Chlor daraus weggenommen 
hal, den eigenthümlichen, so heftigen Geruch des Cyanchlorids, welches davon abdestillirt werden kann *).

Schmilzt man Titanwürfel als feines Pulver mil Kalihydrat, so entwickelt sich Ammoniakgas unter Bildung von titansaurem Kali.

Glüht man die zerriebenen Würfel in einem Porzellanrolır in einem Strom von Wasserdampf, so findet, wie schon Regnault beobachtete, eine reichliche Wasserstoffgasentbindung stall, aber leilet man dabei das Gas durch abgekühlles Wasser, so nimınt dieses den Geruch nach Amınoniak und nach Blausäure an, beide aufserdem leicht nachweisbar durch alle ihnen eigenthümlichen Reactionen.

Wendet man zu diesem Versuche dic Würfel unzerrieben an, so behiilt die entstchende Titansäure die Form abgerundeter Würfel. Aber betrachtet man sie bei ungefähr 300facher Vergröfserung, so erkennt man, dals sic aus einer Aggregalion von zum Theil sehr wohl ausgebildeten, glänzenden Krystallen bestehen, und diesc Krystalle haben merkwürdiger Weise die Form des Anatas. Es sind spitze Quadratoctaëder meist mil starker Querstreifung der Flïchen, von starkem diamantähnlichem Glanz, theils farblos, meisl aber von nelkenlbrauner Farbe; kurz es ist künsllicher Analas mit allen Eigenthümlichkciten dieses seltenen Minerals.

Die Feststellung der quantitativen Zusammensetzung der Würfel bot mancherlei Schwierigkeiten dar und veranlafste anfangs manchen fruchtlosen Versuch. Sie wurden zunächst aus

*) Man erhïlt diese Verbindung unmittelbar, wenn man Cyanchloridgas zu Titanchlorid leitet. Die Vereinigung geht unter starker Wärmeentwickclung sehr rasch vor sich. Die Eigenschaften und Zusammensetzung dieses Körpers werde ich an einem andern Orte näher angeben. - Dafs George, der Entdecker des Titanchlorids, dieser Krystalle nicht erwähnt, ist kein Beweis, dafs er sie nicht erhielt, sondern ein Beweis, dals er sie nicht beachtete. 
den Eisen - und Gestellsteinsmassen, worin sie in den Hohöfen vorzukommen pflegen, durch Behandlung mit concentrirter Salzsäurce und Schwefelsäure und Schlämmen ausgeschieden und zuletzl durch concentrirte Flufssäure sorgfällig von fremder Beimengung gereinigt. Aber in dicsem scheinbar reinen $\mathrm{Zu}-$ stamle enthalten sic noch Graphitblättchen mechanisch eingemengt, und selbst auf den anscheinend reinsten bemerkt man unter starker Vergröfserung hier und da noch eisenschwarze Parthien von glänzenden Graphitblätlchen aufsitzend, die nicht leicht zu entfernen sind.

Bei cinem Versuche, wobei 1,4105 Grm. ausgelesener Würfel in luftreiem trocknem Chlorgas verbrannt und bis zur völligen Erschöpfung geglüht wurden, blieb 0,025 oder 1,772 pC. eines lockeren, schwarzen Rückstandes, indem aulser dem liquiden Chlorid zugleich ein starkes Sublimat von krystallisirtem Titanchlorid-Cyanid erhalten wurde. Wasser zog aus dem schwarzen Rückstand 0,012 oder 0,85 pC. eines zerllielslichen Salzes aus, welches aus Chlorcalcium mit einer Spur Chlorkalium bestand. Der unlösliche schwarze Rückstand wog 0,013 oder $0,921 \mathrm{pC}$. und bestand aus mikroskopischen glänzenden Graphitblällchen.

lch war anfangs geneigt, die Chlorcyan-Bildung überhaupt solchem blofs eingemengten Kohlenstoff zuzuschreiben und die eigentliche Substanz der Würfel für blofses Stickstofftitan zu lalten. Allein mchrfache Versuche, durch Erhitzen eines innigen Gemenges von Jem gleich zu beschrcibenden Stickstofftitan mil fein zerricbenem Roheisen-Graphit oder mit Zuckerkohle in Irockncm Chlorgas das so characteristische TitanCyanchlorid hervorzubringen, gaben licine Spur von diesem Körper. Hieraus ging also hervor, dafs dic Würfel zwar ungefähr 1 pC. Graphit als unwesentliche Einmengung, zugleich aber noch Kohlenstoff in Form von Cyan enthalten müssen. 
Wöhler, uiber die Natur des metallischen Titans. $\quad 27$

Zur Bestimmung des Tilangehaltes wurden drei analytische Versuche gemacht :

1) 1,6745 Grm. zerriebener, durch Schlämmen des Pulvers möglichst von Graphit befreiter Würfel wurden in einem Platinschiff in einem langsamen Strom von Sauerstoffgas verbrannt. Die Masse verglinmte mit weifsem.Feucr. Die gebildete Titansäure war in Folge dieser starken Verbrennungshitze sehr zusammengesintert und liefs daher eine unvollständige Verbrennung vermuthen. Sie wurde daher zum zweiten Mal in einem Sauerstoffstrom geglüht, und da sie dadurch an Gewicht noch zugenommen hatle, so wurde sie hierauf fein zerrieben und in einem Platintiegel über der Spirituslampe unter Luftzutrill einer sehr lange dauernden Glïhhitze ausgeselzt, so lange bis sie nicht mehr an Gewicht zunahm. Sie war hell zimmtbraun und wog nun 2,133 Grm., entsprechend 76,58 pC. Titan in den Würfeln *).

2) 2,948 Grm, ausgesuchler, sehr fein zerriebener Würfel wurden bei-slarker Glühhitze in cincm Porzellanrohr auf einem Porzellanschiff in einem lange anhallenden Strom von Wasserdampf oxydirt. Die gebildete Tilansüurc wog 3,764 Grm., entsprechend 76,76 $\mathrm{pC}$. Titan in den Würfeln.

3) $1,00 \mathrm{Grm}$, ausgesuchler ganzer .Würfel wurden in einem Plalinliegel in glühend schmelzendem zweifach-schwefclsaurem Kali aufgelöst. Die Oxydalion geht auf diese Weise sehr rasch unler reichliclicr Enlbindung von schwefliger Säure vor sich, anfanglich unter Abscheidung eines leichten Schaumes von Graphit, der aber nach und nach cbenlalls verschwindet. Die vollkommen weifse Masse wurde in viclem lauem Wasser gelöst, die Lösung mit Ammoniak gefällt, der Nicderschlag vollslündig gewaschen und nach dem Trocknen geglïht. Er wog 1,355 Grm., entsprechend 81,47 pC. Titan. Da hier mit

*) Das Atomgewicht des Titans zu 301,55 genommen. 
Wahrscheinlichkeit ein Rückhalt von Schwcfelsäure und Kali zu vermulhen war, so wurde diese Titansäure mit gröfster Sorgfalt durch Digestion mit concentrirler Schwefelsäure wieder aufgeschlossen, die Masse in Wasser gelöst und wieder mit Ammoniak gefällt und gewaschen. Sie wog nun nach dem Glühen, wobei sie unter Ammoniakgeruch verglimmte und bräunlich wurde, 1,305 Grm., entsprechend 78,46 pC. Titan in den Würfeln.

Das Mittel aus diesen drei Versuchen ist 77,26 pC. Tilan. Zur Bestimmung des Stickstoffgehaltes wurde der Versuch gemacht, sehr fein geriebenes Würfelpulver wie bei einer organischen Analyse mit Natronkalk zu glühen und den Stickstoff als Platinsalmiak zu bestimmen. Allein die Zerselzung ging auf diese Weise nur sehr unvollständig vor sich. Eben so wenig gelang es, das bei der Oxydation der Würfel in Wasserdampf entstehende Ammoniak auf diese Weise vollständig zu bestimmen, offenbar weil in der starken Glühhilze im Porzellanrohr zu viel Ammoniak wieder zersetzt wurde. Die Bestimmung des Stickstoffs als Ammoniak durch Schmelzen mit Kalihydrat mifslang ebenfalls, weil dis Glasgefüfs noch vor der vollständigen Oxydation des Würfelpulvers durch das Kalihydrat durchfressen wurde.

Es wurde daher der Versuch gemacht, den Stickstoff durch Schmelzen mit zweifach-schwefelsaurem Kali frei zu machen und als Gas aufzusammeln, was vollkommen gelang. Ausgesuchle, sehr fein zerriebene Würfel wurden in einer kleinen Retorte durch Zusammenschmelzen mit dem zuvor glïhend geschmolzenen Salz aufgelöst. Der Hals der Relorte war mit Asbest, der mit concentrirter Kalilauge benetzt war, locker ausgefïllt. Zur vollständigen $W^{r}$ egnahme aller Kohlensäure und sclıwefligen Säure wurde er mit einer Röhre verbunden, die mit Stückchen von Kalihydrat gefüllt war. An dieser Röhre befand sich, nach Art der früheren Stickstoflbestimmungsmethode 
bei der organischen Analyse, eine Gay-Luss ac'sche Ableitungsröhre, durch die das Gas in einem graduirten Rohr über Quecksilber aufgesammelt wurde.

$0,376 \mathrm{Grm}$. Würfel gaben 58,5 Cubikcentimeter Stickgas bei $17^{\circ}$ C. und $753^{\mathrm{mm}}$. Druck, $=54,57$ CC. bei $0^{\circ}$ und $760^{\mathrm{mm}}$. Druck, $=0,0688$ Grm. oder $18,30 \mathrm{pC}$. Stickstoff.

Es blieb nun noch die directe Bestimmung des als Cyan in den Würfeln enthaltenen Kohlenstoffs übrig. Bei der einen Titanbestimmung, durch Verbrennung der Würfel in Sauerstoffgas, wurde die gebildete Kohlensäure in einem Kaliapparat aufgesammelt. Von 1,6745 Grm. Würfel wurden 0,200 Koblensäure erhalten, entsprechend $3,26 \mathrm{pC}$. Kohlensloff. Allein da es sich zeigte, dafs bei diesem Versuch die Verbrennung nur unvollständig statlgefunden halle, so hatle diese Zahl nur in sofern Werth, als sie eine weilere Bestäligung war, dafs die Würfel aufser dem Graphit noch chemisch gebundenen Koblenstoff enthalten.

Die Würfel haben die auffallende Eigenschaft, als Pulver mit den Oxyden von Kupfer, Blei und Quecksilber gemengt und erhilzt, mil starker, funkensprühender Feuererscheinung und unter Reduction jener Metalle zu verbrennen. Die Wärmeentwickelung hierbei steigert sich bis zur Weifsglühhilze und ist so grofs und so momentan, dafs selbst das Kupfer in einer Glasröhre zu Kugeln zusamumenschmilzt. Eben so heftig verbrennen sie als Pulver mit chlorsaurem Kali.

Diese leichte Oxydation konnte zu einer sicheren Bestimmung des Kohlenstoffs benulzl werden. Nach mehreren Versuchen zeigte es sich, dafs das Bleioxyd, wegen seiner leichten Schmelzbarkeit, das gceignetste Mittel zur vollständigen Verbrennung war. Es wurde in Gestall von schwach geglühter, halb zersetzter, vollkommen koblensäurefreier Mennige angewendet und, zur Mälsigung der Verbrennung, in sehr grofsem Ueberschuls mil dem sehr feinen Würfelpulver gemengt. Die 
Verbrennung geschah, wie bei einer organischen Analyse, in einem Glasrohr und mit Anwendung zweier Kaliapparate.

$0,802 \mathrm{Grm}$. ausgesuchter Würfel gaben 0,134 Grm. Kohlensäure $=4,56 \mathrm{pC}$. Kohlenstoff, den als Graphit darin enthaltenen Kohlenstoff mit eingerechnet.

Nach diesen Analysen enthalten die Würfel in 100 Theilen, mit Vernachlässigung der kleinen, unwesentlichen, wahrscheinlich variirenden Menge von Calcium - und Kaliumverbindung:

\begin{tabular}{lr} 
Tilan & $\mathbf{7 7 , 2 6}$ \\
Stickstoff & 18,30 \\
Kohlenstoff & 3,64 \\
Graphit & 0,92 \\
\cline { 2 - 2 } & $100,12$.
\end{tabular}

Geht man von dem Tilangehalt aus und nimml das Fehlende als Kohlenstoff und Stickstoff, so machen diese zusammen 22,74 aus, was mit der direct gefundenen gemeinschaftlichen Menge $=22,86$ nahe genug übereinstimmt. Und bestimmt man, nach der gufunlenen Titan - und Kohlenstoffınenge, den Stickstoffgrehalt indirect aus dem Verlust, so beträgt er 18,18, was ebenfalls mit der directen Bestimmung $=18,30$ hinreichend nahe slimmt.

Aus diesen Zahlen geht für die Zusammenselzung der 'Tilanwürfel, nach Abzug des unwesentlichen, eingemengten Grapliigehalles, die Formel $\mathrm{Ti}^{2} \mathrm{~N}+3 \mathrm{Ti}^{3} \mathrm{~N}$ hervor, nach welcher sic in 100 Theilen enthalten müssen :

$\begin{array}{lr}\text { 'Titan } & \mathbf{7 8 , 0 0} \\ \text { Stickstoff } & \mathbf{1 8 , 1 1} \\ \text { Kohlensloff } & \mathbf{3 , 8 9} .\end{array}$

I)as heifst sic bestehen in 100 Theilen aus :

Titancyaniir $\quad 16,21$

Sticksloftitan 83,79 .

Ich holfe, dafs es mir gelingt, diese beiden Verbindungen auch für sich darzuslellen. 
Man könnle vermuthen, dafs die Würfel das $\mathrm{C}^{2} \mathrm{~N}$ in Form von sogenanntem Paracyan enthalten; allein da dieser Körper noch zu wenig genau untersucht ist, als dafs man mit Ueberzeugung an sein Dasein glauben könnte, so würde diese Vorstellungsweise keinen gröfseren Werth haben, als die andere, die dadurch, dafs aus den Würfeln wirklich eine Cyanverbindung hervorgebracht werden kann, viel gröfsere Wahrscheinlichkeit für sich hat.

Was die Bildungsweise dieser Würfel betrift, so halle ich es für unzweifelhaft, dafs sie mit der in den Hohöfen schon so oft bcobachteten Bildung von Cyankalium im Zusammenhang steht. Einige Versuche, die ich in dieser Hinsicht angestellt habe, scheinen diese Annahme vollkommen zu bcstätigen.

Ein Gemenge von wasserfreiem Kaliumeisencyanür und Tilansäure wurde in einem verschlossenen Tiegel über eine Stunde lang einer Hitze ausgesetzt, bei der Nickel schmilzt. Es wurde cine braune, ungeschmolzene, poröse Masse erhalten, aus der Wasser nur noch Spuren von Cyankalium auszog. Bei $300 f a c h e r$ Vergröfserung betrachtet, erkannte man darin überall, untermengt mil metallischem Eisen, ein Netzwerk von kupferfarbenen, slark glänzenden, feinen, kurzen Prismen, die unzweifelhaft aus der Substanz der Würfel bestanden. Bei Behandlung der Masse mil concentrirler Salzsäure wurde das Eisen unter hefliger Wasscrstoffentwickelung aufgelöst, mit Zurücklassung eines braunen Pulvers, sehr ähnlich dem Pulver von zerriebenen Würfeln. Unter dem Mikroskop zeigte es sich als ein Gemenge von kupferfarbenen Nadeln mil einer schwarzen Subslanz, nämlich Kohle. Beim Erhitzen an der Luft verglimmle es zu gelblicher Tilansäure; beim Erhitzen mit Kupferoxyd verbrannte es mit Enlwickelung von Weifsglühhitze, indem das dabei reducirle Kupfer zu Kugeln schmolz. Beim Erhilzen mit Kalihydrat entwickelte es reichlich Ammoniakgas. Beim Erhilzen in Chlorgas gab es Tilanchlorid und dic Krystalle von Titan- 
Cyanchlorid in Menge, unter Zurücklassung von pulveriger Kohle.

Bei einem zweilen Versuch wurde dem obigen Gemenge, um vielleicht zur besseren Ausbildung von Krystallen eine schmelzende Masse zu erhalten, noch eine kleine Menge kohlensaures Kali zugeselzt. Es wurde eine schwarze blasige Schlacke erhallen mit einem grofsen Eisenrcgulus, dessen Oberfläche gestrickt krystallinisch und theilweise mit kupferfarbenem 'Titan umgeben war, von dem sich aber weder im Innern noch in der Schlacke eine weitere Menge fand.

Die bis jetzt bezweifclte Angabe von Zinken*), dafs die Tilanwürfel in selır hoher 'Temperatur flüchtig seyen, habe ich bestäligt gefunden. Einige Gramm reiner Tilanwürfel wurden in einem kleinen lulirten Porzellantiegel, der, ungeben und bedeckt von einer dicken Lage Kohlenpulver, in einem gröfseren hessischen Tiegel stand, ungefälır eine Stunde lang Nickelschmelzhilze ausgeselzt. Die Würffel waren scheinbar unverändert geblieben, sie waren durchaus nicht zusammengesintert, aber etwas heller an Farbe und maller geworden. Unter dem Mikroskop zeigten sich die meisten Flächen matt und kryslallinisch geworden, wie ein von Säure oberflächlich angegriffenes krystallinisches Melall; auch waren die Kanten nicht mehr so scharf wie zuvor. Dabei war die innere Seite des hessischen Tiegeldeckels kupferroth, wie verkupfert, geworden, zum Beweis, dafs sich von den Würfeln ein Theil zu verflüchligen angefangen halle und gasförnig durch die Kohlenlage hindurchgegangen war. Der unglasirte Porzellantiegel war "inwendig und auswendig schwarz geworden. Wahrscheinlich würde bei länger andauernder Hitze eine vollständige Verflüchtigung stattgefunden haben. Auch an den Würfeln, wie sie aus den Hohöfen kommen, sind zuweilen solche malte Flächen zu sehen, wie wenn nach ihrer

*) Pogg. Annal. Bd. XXVIII, S. 160. 
Entstehung durch weilere Einwirkung der Hitze eine Verflüchtigung begonnen hätle.

Die Würfel, die mir zu dieser Unlersuchung dienten, stammten alle aus dem Hohofen zu Rübeland am Hlarz, worin neuerlich, wie Hr. Blumenau schälzt ${ }^{*}$ ), eine Titanmasse von wenigstens 80 Pfund gefunden worden ist. Sie füllten theils gangarlige Spalten in der Quarzmasse des Bodensteins aus, theils waren sie in Massen von metallischem Eisen enthalten. Ich habe nicht Gelegenheit gehabt, Würfel aus anderen Hohöfen, namenllich nicht solche, die in Schlacken vorkommen, zu untersuchen; allein es ist wohl nicht zu zweifeln, dafs sie in der Zusammenselzung immer idenlisch sind.

\section{Stickstoff - Titan.}

So lange die 'Titanwürfel für das reine Titan gehallen wurden, war es, bei der Aehnlichkeit der Farbe, ein verzeillicher Irrhum, auch die zuerst von H. Rose aus dem AmmoniakTitanchlorid dargestellte kupferfarbene Substanz dafür zu hallen, wiewohl ein einziger quanlitativer Verbrennungsversuch gezeigt hätte, dafs man von 100 Theilen davon nicht 166 Titansüure erhäll, wie man erhalien muifste, wäre dieser Körper reines Titan, sondern niclıt ganz 120 Tilansäure, dafs also fast $28 \mathrm{pC}$. darin etwas Anderes sind, als Tilan. Dieser andere Körper ist Stickstoff. In der That, das nach Liebig's Molhode **) aus dem AmmoniakTilanchlorid durch Erhitzen desselben in Ammoniakgas dargestellte 'Titan ist Slickstoff-Titan, zusammengesetzt nach der Formel Tis ${ }^{3} \mathrm{~N}^{2}$. Es ist also in der Zusammenselzung verschieden von dem in den Wïrfeln enthaltenen. Auch erkennt man bei näherer Vergleichung, dafs sie beide in der Farbe wesentlich verschieden sind; bei dem Stickstofltitan ist sie mehr kupferroth, bei den Würfeln hat sie einen starken Stich ins Gelbc. Namentlich ist diese viel

") Diese Aunalen Bd. XI.VII, S. 122.

**) Pogg. Ann. XXI, S. 159. 
Wöhler, über die Natur des metallischen Titans.

gelbere Farbe bei manchen, sehr glänzenden Würfeln deutlich, die vielleicht völlig ohne Luftzutritt erkalteten und darum nicht anlaufen konnten.

Mit Kalihydrat geschmolzen, entwickelt das Stickstoftilan rcichlich Ammoniak, eben so beim Glühen in Wasserdampf. In Chlorgas erhitzt, verbrennt es zu Tilanchlorid, aber selbst in inniger Vermengung mit Kohle bildet es dabei keine Krystalle von Titan-Cyanchlorid, wie schon oben erwähnt wurde.

Zur Bestimmung sciner quantitaliven Zusammenselzung war es hinreichend, den Titangehalt darin durch 0xydation zu Titansäure zu ermilleln und aus dem Verlust den Stickstoff zu berechnen.

Die Verbrennung geschah in eincm Platintiegel über der Spiriluslampe und erforderte zur Vollendung jedes Mal über cine Slunde. Zuerst liefen die Blällchen slahlfarben an und dann verglimmten sie. Dabei zeigle sich die Erscheinung, dafs in einer gewissen Periode der Oxydation die Slückchen mit Geräusch zu zerspringen anfingen, und zwar inmer erst, als die Verbindung schon fast vollständig zu Titansäure oxydirt war und die Hilze verstirkt wurde, so dafs es aussieht, als ob die Erscheinung mit den von meinem ausgezcichnelen Freunde so schön dargelegten Uebergängen der cinen Titansäuremodification in die andere im Zusammenhang stehe. Die gebildete Tilansäure war heifs citrongelb, nach dem Erkalten gelblich-weils, während die auf dicse Weise aus den Würfeln gebildete slels heller oder dunkler zimmlbraun war. Bei 500 facher Vergröfserung zeigte sich die ersterc deutlich kryslallinisch, durchscheinend.

$0,276 \mathrm{Grm}$. in dichten, glänzenden, von der Glasfläche, worauf sie sich gebildet hatten, abgelösten Blältchen gaben $0,334 \mathrm{Gl} \cdot \mathrm{m}$. Tilansäure, entsprechend 72,76 pC. Titan.

$0,2345 \mathrm{Grm}$. in glänzenden Blättchen von anderer Darslellıng gaben 0,2810 Titansäure $=\mathbf{7 2 , 0 2}$ Titan. 
0,646 Grm. als dunkel kupferfarbenes Pulver gaben 0,773 Titansäure $=71,94$ Titan.

Das Mittel aus diesen deei Zahlen ist 72,24 pC. Titan, also 27,76 pC. Sticksloff.

Diefs entspricht der Formel $\mathrm{Ti}^{3} \mathrm{~N}^{2}$, nach wolcher dieses Slickstofflitan in 100 Theilen enthalten mufs :

$$
\begin{array}{ll}
\text { Tilan } & \mathbf{7 2 , 1} \\
\text { Slickstoff } & \mathbf{2 7 , 9} .
\end{array}
$$

Diese ist aber nicht die einzige, isolirt darstellbare Verbindung zwischen Stickstoff und Titan; ich habe gefunden, dafs es deren noch zwei andere giebt. Alle diese Verbindungen zeigen, wie die Würfel, die eigenthümliche Erscheinung, als Pulver mit leicht reducirbaren Metalloxyden vermischt und zum Glühen erhilzt, sich unler heftiger, sprühender Feuerentwickelung zu oxydiren und das andere Metall zu reduciren. Alle vertragen eine mindestens bis zur Kupferschmelzhitze gehendc Temperatur, ohne zerselzt zu werden.

Das Sticlisloff-Titan Ti $\mathrm{A}^{*}$ ) entsteht, wenn man Titansäure bei starker Glühhitze einem Strom von trocknem Ammoniakgas aussetzt und darin erkalten läfst. Die Verbindung ist ein dunkel violettes Pulver mit einem Slich ins Kupferfarbene, wie Pulver von sublimirtem Indigo. Wendet man ganze Stücke von Tilansäure an, so erhält man die Verbindung in dunkelviolelt kupferfarbenen, metallisch glänzenden Slücken. Allein die Verwandlung bleibt dann gewöhnlich nur oberflächlich. Selbst Krystalle von Rutil werden auf dicse Weise an der Oberfläche dunkel kupferroth, im Innern schwarz.

$0,549 \mathrm{Grm}$. durch Glühen an der Luft oxydirt, gaben 0,591 'Titansäure. Diefs giebt :

*) Um das schlecht lautende Wort Stickstoff in Zusammensetzungen zu vermeiden, möchte es wohl am besten seyn, die Stickstoffmetalle in Zukunft Nitrele (von Nitretum) zu nennen, also zu sagen Titannitret etc. 
Wöhler, über die Natur des metallischen Titans.

$\begin{array}{lcc} & \text { gefunden, } & \text { berechnet nach Ti } \\ \text { Titan } & 64,66 & 63,269 \\ \text { Slickstoff } & 35,34 & 36,731 .\end{array}$

Der Mangel an besserer Uebereinslimmung hat darin seinen Grund, dafs diese Verbindung beim längeren Glühen in Aınmoniakgas oder in Wasserstoffgas, welches letztere bei ihrer Bildung durch partielle, in der Hilze für sich erfolgende Zorselzung von Ammoniak frei wird, anfängt, Slicksloff zu verlieren und sich in die folgende Verbindung zu verwandeln. Nach dem Gliben in Wassersloffyas gab eine Portion 65,95 Titan, und nach nochmaligem Glühen in Ammonialkgas eine andere 66,6 .

Das Stickstoff - Titan $\mathrm{Ti}^{5} \mathrm{~A}^{3}$ entstcht, wenn man die Verbindung $\mathrm{Ti}^{3} \mathrm{~N}^{2}$ in einem Strom von getrocknetem Wasserstoffgas einer heftigen Glühhilze ausselzt und in dem Gasstrom erkalten läfst. Noch unter der Glühlitze beginnt der Theil vom Slickstoff, den die Verbindung verliert, in Form von Ammoniak wegzugehen, was also ein neuer Fall von Ammoniakbildung ist. Hat man das kupferrothe Slickstoff-'Titan in glänzenden Blällern angewendet, so erhält man die neue Verbindung in schön messinggelben, fast goldfarbenen, slark metallglänzenden Blällchen. Pulverförmig ist sie bronzefarben, melallisch schimmernd.

0,3665 Grm. gaben beim Verbrennen 0,452 Titansäure.

$0,213 \mathrm{Grm}$. von anderer Darstellung gaben 0,262 Titansäure. Diefs giebt :

$\begin{array}{lccc} & \text { I. } & \text { II. } & \text { berechnet nach } \mathrm{Ti}^{5} \mathrm{~N}^{3} \\ \text { Titan } & \mathbf{7 4 , 1 3} & \mathbf{7 3 , 9 4} & \mathbf{7 4 , 1 6} \\ \text { Stickstoff } & \mathbf{2 5 , 8 7} & \mathbf{2 6 , 0 6} & \mathbf{2 5 , 8 4} .\end{array}$

Dieselbe Verbindung scheint zu entstehen, wenigstens der Farbe nach zu urtheilen, wenn man Titansäure in eincm Strom von Cyangas oder von Blausäuredampf glïht. In beiden Fällen erhält man einen melallglänzenden Körper, ungefähr von der Farbe von Glockenmetall, aber innig gemengt mit Kohle, welche wohl auch die Abweichung in der Farbe bedingt. Bei der Dar- 
stellung mit Blausäuredampf bildele sich Cyanammonium und die Stückchen waren meist mit rulsartiger Kohle bclegt. Bei der mit Cyangas dargestellten dagegen war keine Kohle sichtbar. Sie kam aber zum Vorschein und blieb zurück, als die Verbindung in Chlorgas verbrannt wurde. Hierbei bildete sich nur Titanchlorid und keine Spur der krystallisirten Cyanidverbindung, zum Beweise, dafs dieses Stickstofl-Titan das in den Würfeln enthaltene Cyantitan nicht enthält. Auffallend ist es indessen, dafs es auf diesem Wege nicht entsteht.

Aus dem nun Angeführten geht hervor, dafs es vier, in Eigenschaften und Zusammensetzung verschiedene Verbindungen zwischen Stickstoff und Titan giebt; ich halte es aber für sehr wahrscheinlich, dafs hicr ein ähnliches Verhältnifs stattindet, wie bei den $0 x y$ dationsstufen mancher Metalle, dafs nämlich nur zwei davon selbstständige Verbindungsstufen sind, die beiden anderen aber Verbindungen zwischen diesen. Als die einfachen Stickstoffverbindungen des Titans könnlen betrachtet werden das in den Würfeln enlbaltene, freilich für sich noch nicht dargestelle Stickstoff-Titan $\mathrm{Ti}^{3} \mathrm{~N}$, und zweilens das violett kupferfarbene, welches durch Glühen der Titansäure in Ammoniakgas gebildet wird, = Ti N. Dic beiden anderen könnten Verbindungen zwischen jenen beiden ersteren seyn, wie die folgende Aufstellung zeigt :

Die Würfel . . . . . $=\mathrm{Ti} \mathrm{Gy}+3 \mathrm{Ti}^{3} \mathrm{~N}$.

Das violelle $: \cdot .:=0$

Das goldfarbene, $\quad \mathrm{Ti}^{5} \mathrm{~N}^{3}=2 \mathrm{Ti} \mathrm{N}+\mathrm{Ti}^{3} \mathrm{~N}$.

Das kupferfarbene, $\mathrm{Ti}^{3} \mathrm{~N}^{2}=3 \mathrm{Ti} \mathbf{N}+\mathrm{Ti}^{3} \mathrm{~N}$.

Es bleibt mir nun noch die Frage zu beantworten übrig, wie eigenllich das reine metallische 'Fitan beschaffen ist. Dieses ist zuerst von B e r z cli us dargestellt, wiewohl nicht näher untersucht worden. Es ist der schwarze Körper, den er durch Erhitzen von Kaliumlitan-Fluorür mil Kalium erhielt *). Ich habe

*) Pogg. Annal, IV, S. 3. 
es auf diese Weise dargestellt, indem ich die unter starker Feucrerscheinung stattfindende Reduction in einem bedeckten Platintiegel uiber der Spiriluslanpe vornalın. Die erkaltete Masse wurde mil vielem Wasser übergossen, die leichteren, litansäurehalligen, grauen Antheile sorgfältig abgeschlämmt und das sclıwere Pulver zuletzt, zur Entfernung von allem unzersetzt gebliebenen Salz, mit vielem lauen Wasser gewaschen und getrocknel.

Das metallische 'Titan ist ein dunkelgraues, unkrystallinisches Pulver, sehr ähnlich dem bei gelinder Hilze durch Wasserstoffgas reducirten Eisen. Bei 100facher Vergröfserung sieht man, dafs es aus zusammengesinterten Klumpen besteht und vollkommnen Metallglanz und die Farbe des Eisens hat. Auch durch Druck nimmt es keine Spur von Kupferfarbe an. Beim Erhitzen an der Luft verbrennt es mit einer aufserordentlich glänzenden Feuererscheinung. In eine Flanme gestreut, verbrennt es, noch hoch über derselben, mil demselben blendenden Glanz und demselben Funkensprühen, wie das Uran. Das kleinste, kaum sichtbare Stäubchen bildet einen äufserst glänzenden, sternförmigen Funken. Mit Mennige oder Kupferoxyd vernischt und exhitzt, verbrennt es mit so heftiger Feuerentwicklung, dafs die Masse wie ein Schufs spriihend aus der Röhre herausgeschlendert wird. In Sauerstoffgas erhilzt, verbrennt cs momentan mit blendendem, blitzähnlichem Feuer. Die entstehende Titansäure ist pulverig, aber bei starker Vergröfserung sieht man, dafs sie zusammengesintert, glänzend und krystallinisch ist und hier und da metallischglänzende, eisengraue Kugeln eingeschmolzen enthält, die ohne Zweifel Titan sind, wclches, bei der so momenlan stallfindenden Verbrennung der Oxydation entgehend, geschmolzen ist. Ich glaube nicht, dafs es noch einen anderen Körper giebt, der mit so aufserordentlicher Entwickelung von Licht und Wärme verbrennt, wie das Titan. Aehnlich glänzend ist scine Verbrennung in Chlorgas, welches übrigens bei gewöhnlicher 'Temperalur darauf nicht wirkt. 
Das Titan ist ein wasserzersetzendes Metall, womil auch die von H. Rose und Regnault bcobachtete wasserzerselzende Eigenschaft des Schwefellitans in Einklang steht. Schon bei $100^{\circ}$ fängt es für sich an das Wasser zu zersetzen und schwach Wasserstollgas zu entwickeln. Von Salzsâure, jedoch erst beim Erwärmen, wird es unler lebhafter Wasserstoffentbindung aufgelöst. Die Auflösung ist farblos und enthält wahrscheinlich das Chlorïr, Ti Gl. Ammoniak bildet darin einen schwarzen Niederschlag, wahrscheinlich von Oxydhydrat, welcher aber beim Erwärmen sogleich beginnt Wasserstoffgas zu entwickeln und blau zu werden, wahrscheinlich titansaures Tilanoxyd, welches dann bald in weifse Titansäure übergeht.

Was endlich das von Laugier, Berthier u. A. beschriebene, angeblich metallische Titan ist, welches sie durch Reduction von Tilansäure in Kohlentiegeln bei hefligem Essenfeucr erhielten und theils als messinggelb, theils als kupferroth beschreiben *), lasse ich unausgemacht, glaube aber nicht, dafs es metallisches Titan war, man müfste denn bei diesem Körper zweierlei allotropische Zustände annehmen wollen.

Die obigen Erfahrungen werde ich nun auch auf andere, dem Titan verwandte Körper, namentlich auf Wolfram, Silicium, Bor u. s. w. auszudehnen versuchen, in der Hoffnung, dadurch überhaupt unsere Kenninisse von den Stickstoffmelallen, deren erste Entdeckung man Schrötter verdankt, zu erweitern und auch über die von Balmain enldeckten Verbindungen sicheren Aufschlufs zu erhalten.

Dem Hrn. Dr. Städeler sage ich meinen Dank für die grofse Hülfe, die er mir bei dieser Arbeil zu leisten die Gefälligkeit hatle.

*) Gmelin's Handbuch 1848, II, S. 431. 